\title{
Ocular and cerebral trauma in non-accidental injury in infancy: underlying mechanisms and implications for paediatric practice
}

\author{
M A Green, G Lieberman, C M Milroy, M A Parsons
}

\begin{abstract}
Aims-To determine the sites, mechanisms, and clinical significance of injuries to the eyes and brains of children with non-accidental injuries in relation to differing levels of trauma.

Methods-A forensic pathological study of injuries in the eyes and brains of 23 consecutive children dying of non-accidental injuries over a 4 year period (1988-92) under the jurisdiction of Yorkshire and Humberside coroners.

Results-Sixteen children died from cerebral injuries and seven died from noncerebral causes. There were high incidences of retinal detachment $(63 \%)$ and subhyaloid (75\%), intraretinal (75\%), and perineural $(68 \%)$ haemorrhages in CNS deaths. Local subhyaloid haemorrhages and retinal detachment were more common at the periphery and optic disc than at the equator. There was a strong correlation between CNS and eye trauma scores in all 23 children $(r=0.7551$, $p<0.0001)$. Ranking of injuries by severity suggests progressively more trauma required for (a) subdural haemorrhage, (b) subhyaloid, intraretinal, perineural haemorrhages, and (c) retinal detachment. At highest trauma levels choroidal and vitreous haemorrhages were associated with additional cerebral lacerations, intracerebral and subarachnoid haemorrhages.
\end{abstract}

Conclusions-In non-accidental (and probably accidental) infantile head injury the earliest eye injuries (coinciding with subdural haemorrhage) could be missed if indirect ophthalmoscopy is not performed. Retinal detachment and multiple (particularly choroidal/vitreous) haemorrhages may indicate additional cerebral lacerations and/or intracerebral haemorrhage. Vitreous traction is the likely cause of intraocular pathology.

(Br f Ophthalmol 1996; 80: 282-287)

Retinal lesions associated with head injury were described first by Purtscher in 1910, ${ }^{1}$ and in 1946 Caffey noted in infants the association of subdural haematomas, long bone injuries, and retinal haemorrhages with minimal or absent signs of external trauma. ${ }^{2}$ Physical child abuse was first described by Tardieu as long ago as $1860,{ }^{3}$ but in $1962 \mathrm{Kempe}^{4}$ coined the term 'battered child syndrome', and later Caffey described injuries associated with shaking infants in 1972,5 and used the term 'whiplash shaken infant syndrome' in $1974 .^{6}$ Since these early reports numerous ocular injuries have been recognised as common in non-accidental injury to children, including periorbital haematoma, eyelid laceration, subconjunctival haemorrhage, subluxed or dislocated lens, cataracts, glaucoma, anterior chamber angle regression, iridodialysis, retinal dialysis or detachment, intraocular haemorrhages, optic atrophy, and papilloedema (reviewed by Kaplan ${ }^{7}$ and Morris ${ }^{8}$ ). Lesions may be multiple, and at different stages from separate episodes of trauma.

Retinal haemorrhages are a frequent finding in childhood non-accidental injury, and Eisenbrey ${ }^{9}$ and Guilland et al ${ }^{10}$ regard such haemorrhages as diagnostic of child abuse when accompanied by intracranial injuries in the absence of a verifiable history of severe accidental head injury. The exact pathogenesis of these haemorrhages is disputed. Several mechanisms have been postulated, including direct tracking of blood from intracranial bleeding, ${ }^{11}$ haemorrhage secondary to raised intracranial pressure, ${ }^{12}$ and retinoschisis (splitting) related to vitreous traction forces. ${ }^{13}$ The precise method by which trauma is induced has also been disputed, and Duhaime et al ${ }^{14}$ considered that impact of the head with solid surfaces was required in addition to shaking of the child. However, it is now increasingly accepted that shaking alone can cause the cerebral injuries, ${ }^{15}$ and that rotational forces and whiplashing affect the child's head during shaking, ${ }^{16}$ and on this basis a campaign has been launched to educate parents and other carers about the dangers of shaking infants. ${ }^{17}$

We initiated our study of ocular and cerebral injuries in infants who died as a result of nonaccidental injury to determine whether it would be possible, by analysis of the precise sites and extent of injuries, and by ranking patients in order of severity of traumatic

Key points

In non-accidental (and probably accidental) head injury in infants:

- the earliest intraocular injuries are local subhyaloid haemorrhage and local retinal detachment; these may be peripheral and missed without indirect ophthalmoscopy

- if ANY intraocular injury is present subdural haemorrhage is very likely (and can develop later)

- retinal detachment and multiple haemorrhages (particularly choroidal or vitreous) may indicat additional cerebral lacerations or intracerebral haemorrhage 
lesions, to determine both the relative levels of trauma required for each injury and the order in which injuries are sustained with increasing head trauma. As pathologists we felt that this approach was most likely to produce information about the pathogenesis of individual cerebral and ocular lesions in non-accidental injury and accidental head injury. We also hoped that by ranking cerebral and ocular injuries in order of severity we might be able to demonstrate associations between, for example, ocular clinical signs and specific cerebral injuries which would be useful in clinical paediatric practice.

\section{Subjects and methods}

We examined the detailed files of all children up to 3 years old who had died under the jurisdiction of the Yorkshire and Humberside coroners during the 4 year period 1988-92. We initially selected 25 children for this study whose death was attributable to unequivocal non-accidental injury, determined from reviewed autopsy findings, witness statements, and court transcripts of evidence to establish the circumstances surrounding each death. A full and very detailed 'forensic' necropsy had been performed in all cases by members of the Department of Forensic Pathology in Sheffield and Leeds (departments amalgamated into the Department of Forensic Pathology of the University of Sheffield in 1991; the department examines all cases of suspicious death occurring in Yorkshire and Humberside). Twenty three of the original 25 children dying of non-accidental injury had eyes removed for examination, and these 23 cases are the subjects of this study.

The eyes from all 23 children with nonaccidental injury were examined initially in situ after removal of the roof of the orbit, to determine the extent of orbital and periocular traumatic abnormalities, and to establish the extent of perineural (subdural) haemorrhage into the optic nerve sheath. The eyes were then removed, using the minimum of trauma, and fixed in formaldehyde, then alcohol. After fixation we examined the eyes externally before standard removal of superior and inferior calottes, embedding in paraffin wax, and preparation of five horizontally orientated haematoxylin and eosin sections through the centres of the optic nerve and pupil, and one additional section of each of Perls' Prussian blue for haemosiderin and Martius scarlet blue for fibrin. We recorded traumatic ocular lesions by determining the presence $(+)$ or absence (0) in one or both eyes of retinal detachment (using standard histological criteria, and excluding artefactual retinal detachments attributable to formalin fixation or postmortem trauma); and vitreous, subhyaloid, intraretinal, choroidal, and perineural (optic nerve) haemorrhages. These findings were added to produce an eye trauma score, with and without retinal detachment ('total eye trauma' and 'haemorrhage' 'scores respectively). Table 1 was produced by placing children into groups with and without retinal detachment, then ranking them according to total eye trauma scores. Where eye trauma scores are the same, ranking was performed by deeming choroidal haemorrhage more severe (more trauma required) than vitreous or subhyaloid haemorrhage.

We recorded the presence and extent of central nervous system (CNS) traumatic lesions by noting the presence $(+)$ or absence (0) of subdural, subarachnoid and intracerebral haemorrhage, primary axonal injury, and cerebral lacerations, with very extensive haemorrhages scoring ' ++ '. We produced an 'intracranial trauma score' by addition of these individual injury scores.

In order to determine the precise position of intraocular lesions, we noted (in five sections per eye) the presence or absence of retinal detachment and subhyaloid haemorrhage at five points between the ora serrata (the point at which the peripheral retina meets the pars plana of the ciliary body) anteriorly and the optic disc posteriorly, using a microscopelinked video camera and Archimedes 420/1 computer with 'Digit' image analysis. Results were plotted as the percentage of positive findings at each of five relative retinal distance points.

We performed statistical analysis of trauma scores between groups of patients using the non-parametric Mann-Whitney test. We used Fisher's exact test to determine the significance of different incidences of specific traumatic lesions between different groups, and the nonparametric Spearman rank correlation method to quantitate the degree of linear association between CNS and ocular trauma scores. All $p$ values are two tailed/two sided, and results were deemed 'significant' at $p$ values of 0.05 or less.

\section{Results}

Of the 25 children under the age of 3 years who died of non-accidental injury under the jurisdiction of the Yorkshire and Humberside coroners in the period 1988-92, two were excluded from the study because the eyes had not been removed for pathology. Death was attributable to head injury in $16(70 \%)$ of the 23 subjects of this study; the remaining seven $(30 \%)$ died of non-accidental injuries other than head injury (including asphyxia, abdominal injuries, etc).

Table 1 illustrates the range and extent of traumatic eye injuries and central nervous system (CNS) injuries in the 16 subjects who died as a result of head injury, and the single patient with ocular lesions from the group of seven whose deaths were a result of injuries other than head injury (the six subjects in the latter group without CNS or ocular lesions are not shown in this table). The mean age at death was 6.9 months, with a range of 0.5 to 24 months.

All but one of the 16 infants whose death was attributed to head injury had subdural haemorrhage (94\%); in eight of these $(53 \%)$ subdural haemorrhage was the only localised CNS injury, but the remaining seven children 
Table 1 Ocular and intracranial trauma in non-accidental injury in infants

\begin{tabular}{|c|c|c|c|c|c|c|c|c|c|c|c|c|c|c|c|c|c|}
\hline \multirow[b]{2}{*}{ 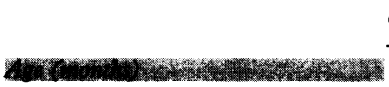 } & \multicolumn{10}{|c|}{ Group $A$} & \multicolumn{6}{|c|}{ Group B } & \multirow{2}{*}{$\frac{\text { Group } C^{\star}}{10}$} \\
\hline & 1 & 24 & 7 & 4 & 2 & 20 & 1 & $2 \cdot 5$ & 4 & 3 & 2 & 10 & 24 & $0 \cdot 5$ & 2 & 1 & \\
\hline 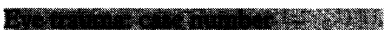 & & 5 & 7 & 13 & 4 & 12 & 10 & 11 & 14 & 8 & 9 & 2 & 3 & 15 & 16 & 17 & 6 \\
\hline Vitreous haemorrhage & 0 & 0 & 0 & 0 & $4 \times 4=2$ & 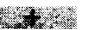 & 0 & 0 & 0 & 0 & 0 & 0 & 0 & 0 & 0 & 0 & + \\
\hline Subhyaloid haemorrhage & toit & & & & & & & & 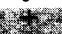 & 0 & rexts & 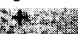 & $1+9$ & 0 & 0 & 0 & + \\
\hline Intraretinal haemorrhage & & & & & & 3. & & & & 0 & $m_{1} x$ & $64 ?$ & at: & 0 & 0 & 0 & + \\
\hline Choroidal haemorrhage & t. & 83 & 5 & 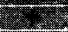 & 0 & 0 & 0 & 0 & 0 & 0 & $x+2$ & 0 & 0 & 0 & 0 & 0 & 0 \\
\hline Perineural haemorrhage & Fe & ches & $\sqrt{2}$ & (28 & & & & & & 0 & $5 \times 4 \times$ & $2+2=4$ & 0 & 0 & 0 & 0 & 0 \\
\hline Total eye trauma score & 5 & 5 & 5 & 5 & 5 & 5 & 4 & 4 & 4 & 1 & 4 & 3 & 2 & 0 & 0 & 0 & 3 \\
\hline It & & 7 & 3 & 3 & 4 & 6 & 1 & 3 & 3 & 0 & 1 & 1 & 2 & 2 & 1 & 1 & 0 \\
\hline Subdural haemorrhage & + & ++ & ++ & + & ++ & ++ & + & ++ & ++ & 0 & + & + & ++ & ++ & + & + & 0 \\
\hline Subarachnoid haemorrhage & 0 & + & 0 & ++ & 0 & ++ & 0 & + & 0 & 0 & 0 & 0 & 0 & 0 & 0 & 0 & 0 \\
\hline Intracerebral haemorrhage & 0 & ++ & 0 & 0 & ++ & + & 0 & 0 & + & 0 & 0 & 0 & 0 & 0 & 0 & 0 & 0 \\
\hline Primary axonal injury & 0 & + & 0 & 0 & 0 & 0 & 0 & 0 & 0 & 0 & 0 & 0 & 0 & 0 & 0 & 0 & 0 \\
\hline Cerebral lacerations & 0 & + & + & 0 & 0 & + & 0 & 0 & 0 & 0 & 0 & 0 & 0 & 0 & 0 & 0 & 0 \\
\hline $\begin{array}{l}\text { Shaking } \\
\text { Shaking/beating } \\
\text { Asphyxia }\end{array}$ & + & + & + & + & + & + & + & + & + & + & + & + & + & + & + & + & + \\
\hline
\end{tabular}

Traumatic eye and intracranial injuries, modes of injury, and ages of infants dying of non-accidental injury in Yorkshire and Humberside in the period $1988-92$. The notations ' + ' and ' ++ ' indicate the presence and severity of injuries, and constitute eye or CNS trauma scores of 1 and 2 respectively. Patients are in three groups in order of cause of death and severity of intraocular trauma and aetiology of the lesions. Infants in groups A and B have died as a result of cerebral injury, and are divided according to presence (group A) or absence (group B) of retinal detachment. Group C consists of seven infants who died of injuries other than cerebral trauma.

^Six patients in group C (cases 18-23 inclusive) have neither cerebral nor eye trauma, and are not included in this table. The illustrated group C case of nonaccidental injury was due to asphyxia, and ocular haemorrhages were present. See text for further explanations.

(47\%) had one or more additional CNS injuries which included subarachnoid haemorrhage, intracranial haemorrhage, and cerebral lacerations. When we compared the groups of children dying from head injury with deaths from other causes there was a very strong association between head injury death and the presence of subdural haemorrhage $(p<0.0001$, Fisher's exact test), but not with the presence of the other recorded traumatic CNS lesions. The presence or absence of cerebral oedema was difficult to assess with any accuracy from the pathological findings which we were able to review, and for this reason was not recorded. In one case (case 8), however, cerebral oedema was recorded as the only demonstrable cerebral abnormality secondary to shaking of the infant. We found no evidence of scalp haematomas or soft tissue contusions on the head in any of the children who died from CNS injury.

There is a strong association between CNS injury and traumatic ocular lesions. Eye injuries were present in $81 \%$ of the 16 children who died of head injuries but only $17 \%$ (one of seven) of those whose death was due to other causes ( $p<0 \cdot 005$, Fisher's exact test). Children who died of CNS injuries had a significantly higher 'total' eye trauma score (3.25 (SD 0.50) versus $0.43(0.43), \mathrm{p}<0.004)$ and 'haemorrhage' score $(2.63(0.42)$ versus $0.43(0.43)$, $\mathrm{p}=0.01$ ) than those without head injury
(Mann-Whitney test). We also found a very strong positive correlation in all 23 children between CNS trauma scores and both 'total' eye trauma scores (Spearman rank correlation, $r=0.7551, \mathrm{p}<0.0001$ ), and 'haemorrhage' scores (Spearman rank correlation, $r=0.7254, \mathrm{p}<0.0001$ ).

The incidences of subhyaloid, intraretinal, and perineural (optic nerve) haemorrhages were significantly increased in children who died as a result of CNS trauma, and in those with subdural haemorrhage (Table 2). Although all of the children with perineural (subdural) optic nerve (sheath) haemorrhages had co-existing subdural haemorrhages, the perineural haemorrhages involved only the distal segment of the nerve, and did not involve the proximal part of the optic nerve within the apex of the orbit, confirming that there was no direct connection between these two separate sites of haemorrhage. Although the incidence of retinal detachment was significantly increased in children dying with CNS injury (Table 2), the increased incidence of retinal detachment in those with subdural haemorrhage alone was not quite significant $(p=0 \cdot 074)$.

We were interested to determine whether there was a relation between retinal detachment and CNS injury and other eye injuries in the children who had died of CNS trauma. There were proportionally more individual

Table 2 Specific traumatic eye injuries in infants dying of central nervous system injuries in non-accidental injury

\begin{tabular}{|c|c|c|c|c|c|c|}
\hline \multirow[b]{2}{*}{ Eye injuries } & \multicolumn{3}{|c|}{ CNS deaths } & \multicolumn{3}{|c|}{ Subdural haemorrhage } \\
\hline & $\begin{array}{l}\text { No } \\
(n=7)\end{array}$ & $\begin{array}{l}\text { Yes } \\
(n=16)\end{array}$ & $\begin{array}{l}\text { Statistical } \\
\text { significance }\end{array}$ & $\begin{array}{l}\text { No } \\
(n=8)\end{array}$ & $\begin{array}{l}\text { Yes } \\
(n=15)\end{array}$ & $\begin{array}{l}\text { Statistical } \\
\text { significance }\end{array}$ \\
\hline $\begin{array}{l}\text { Retinal detachment } \\
\text { Vitreous haemorrhage } \\
\text { Subhyaloid haemorrhage } \\
\text { Intraretinal haemorrhage } \\
\text { Choroidal haemorrhage } \\
\text { Perineural haemorrhage }\end{array}$ & $\begin{array}{l}0 \\
0 \\
1(14 \%) \\
1(14 \%) \\
0 \\
0\end{array}$ & $\begin{array}{l}10(62 \cdot 5 \%) \\
2(12 \cdot 5 \%) \\
12(75 \%) \\
12(75 \%) \\
5(31 \cdot 3 \%) \\
11(68 \cdot 8 \%)\end{array}$ & $\begin{array}{l}\mathrm{p}<0.05 \\
\mathrm{NS} \\
\mathrm{p}<0.02 \\
\mathrm{p}<0.02 \\
\mathrm{NS} \\
\mathrm{p}<0.005\end{array}$ & $\begin{array}{l}1(12.5 \%) \\
1(12.5 \%) \\
1(12.5 \%) \\
1(12.5 \%) \\
0 \\
0\end{array}$ & $\begin{array}{r}9(60 \%) \\
2(13 \%) \\
12(80 \%) \\
12(80 \%) \\
5(33 \%) \\
11(73 \%)\end{array}$ & $\begin{array}{l}\text { NS } \\
\text { NS } \\
p<0.006 \\
p<0.006 \\
\text { NS } \\
p<0.002\end{array}$ \\
\hline
\end{tabular}

There is a significant association between subhyaloid, intraretinal, and perineural optic nerve haemorrhage and death due to both central nervous system (CNS) injury and subdural haemorrhage. Retinal detachment is significantly increased in association with death due to CNS injury, but its increased incidence in subdural haemorrhage is not quite significant statistically $(p=0.074)$ (Fisher's exact test, two sided $p$ values). NS = not statistically significant $(p>0.05)$ 
CNS injuries in the whole group of subjects with retinal detachment $(42 \%)$ than in the group of those with CNS injury but no retinal detachment (25\%) (Table 1), but this is not quite statistically significant $(p=0.533$, Fisher's exact test. NB, ' ++ ' counted as ' + ' for this calculation). Similarly, although the 'CNS trauma' score and 'haemorrhage' score are higher in subjects with retinal detachment than in those without $(3.1(0.69)$ versus $1.33(0 \cdot 21)$, $\mathrm{p}=0.093$ and $3.3(0.40)$ versus $1.50(0.72)$, $\mathrm{p}=0.056$ respectively), this was not quite statistically significant. There were no statistically significant differences between incidences of the individual intracranial injuries listed in Table 1 and presence or absence of retinal detachment. There were proportionally more individual 'haemorrhagic' ocular injuries (shaded areas in Table 1) in the whole group of subjects with retinal detachment $(66 \%)$ than in the group of those without retinal detachment $(66 \%$ versus $30 \%$ respectively, $\mathrm{p}<0.003$, Fisher's exact test). Although there are much higher proportions of subjects with individual 'non-detachment' eye injuries with retinal detachment than without detachment (Table 1, shaded areas) the numbers are small, and perineural (optic nerve) haemorrhage is the only individual lesion with a statistically significantly increased incidence in children with retinal detachment than without detachment $(90 \%$ versus $33 \%$ respectively, $p<0.04$, Fisher's exact test).

\section{SITES OF LESIONS}

Figure 1 shows the incidence and relative retinal sites of true (non-artefactual) retinal detachment and subhyaloid haemorrhage in the 10 and 12 subjects, respectively, with these lesions. The incidence of both of these lesions is lowest at the equator of the eye, but is almost doubled at the periphery of the retina/ora serrata, and is increased (but less so) posteriorly around the optic disc and in relation to the macula.

\section{Discussion}

We believe that this study of infants with nonaccidental injury, the first to combine the identification of the nature and precise site of ocular injuries with quantitation of the extent of injuries in the CNS and eye, provides new information which indicates the mechanism of the injury, and has important implications for clinical paediatric practice.

It is important to place the extent and limitations of this study in context. Our findings are based on the study of 23 consecutive infants with non-accidental injury in a 3 year period, 16 of whom died of central nervous system trauma and seven of whom died of injuries to other areas of the body. We felt that it was vital to the accuracy of the study to exclude cases where the diagnosis of non-accidental injury was in any doubt, and also to exclude cases where there was no opportunity for either histological examination of the eyes, or pathological examination of the CNS. The

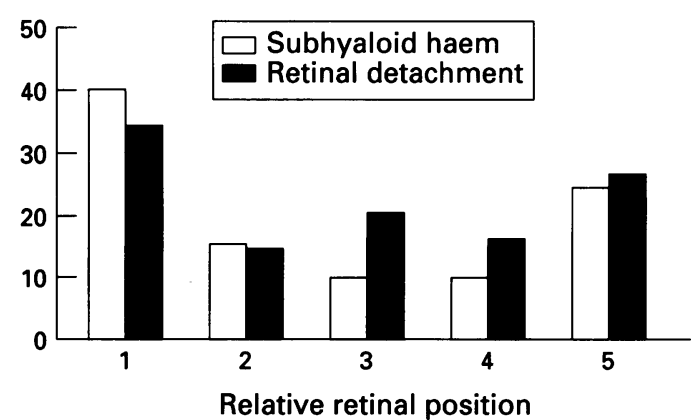

Figure 1 Relative positions of retinal lesions (subhyaloid haemorrhage and local retinal detachment) in infants dying of non-accidental head injury. The incidence of these lesions (\% positive findings in five sections per eye) is highest anteriorly in the peripheral retinalora serrata (position 1), next highest posteriorly at the disc/macula (position 5), and lowest at the equator (position 3). This suggests that traction by the vitreous at the sites of its attachment, the ora serrata and the optic disc, causes the injuries at these sites.

study is also biased towards more serious ocular and CNS injuries, as 'survivors' of nonaccidental injury (with, presumably, less serious injury) are excluded. We believe, however, that we have obtained valid results from this study by statistical analysis of non-selected consecutive cases of unequivocal non-accidental injury deaths in infancy.

It is clear that all except one of the infants who died as a result of head injury had subdural haemorrhages which ranged from small to extensive, and this finding is in keeping with many previous studies. ${ }^{6} 10111415$ The single case (case 8 ) without subdural haemorrhage, however, died of documented cerebral oedema alone, and it is likely that cerebral oedema was present as an important factor in many 'head injury' deaths in our study, by analogy with high incidences reported by Munger et al ${ }^{18}$ and Bundenz et al. ${ }^{19} \mathrm{It}$ is interesting to note that subarachnoid haemorrhages, intracerebral haemorrhages, and cerebral lacerations were never present without coexisting subdural haemorrhages in the infants with head injury in this series, although $63 \%$ of the infants had subdural haemorrhage as the only localised intracranial injury. This suggests that less trauma is required for the development of subdural haemorrhage than for subarachnoid and intracerebral haemorrhage or cerebral laceration, a theory in keeping with the known pathogenesis of these injuries in adults. Subarachnoid haemorrhage, intracerebral haemorrhage, and cerebral lacerations were found only in the group of patients with retinal detachment. Infants with retinal detachment also had a significantly higher incidence of both perineural haemorrhage and total 'nondetachment' ocular injuries, and this supports the supposition that more trauma is required for intracranial traumatic lesions other than subdural haemorrhage. All patients with perineural (subdural) optic nerve haemorrhage had coincident intracranial subdural haemorrhages, although these were also present in four of the five patients without perineural optic nerve haemorrhage. Examination of only the anterior half of the intraorbital optic nerve might lead to the conclusion that these intracranial and perineural subdural 
haemorrhages were related. However, we found, as did Munger et al,,$^{18}$ that there was no perineural optic nerve haemorrhage at the apex of the orbit, and conclude that perineural optic nerve haemorrhage is a separate entity in these children, and not the result of direct tracking of intracranial subdural haemorrhage into the optic nerve sheath.

\section{INTRAOCULAR TRAUMATIC LESIONS}

Our findings indicate that there is a significantly higher proportion of 'whole group' intraocular injuries in infants with retinal detachment than in those without retinal detachment, although perineural optic nerve haemorrhage was the only individual ocular lesion with a statistically significantly increased incidence in this study. A larger study is required to determine whether, as we suspect, there is a relation between the presence of retinal detachment and other individual intraocular injuries, and with CNS trauma scores. The incidences and patient distribution of subhyaloid and intraretinal haemorrhages are very similar to the incidence of perineural optic nerve haemorrhage but, because they are both present in one more patient without retinal detachment, this is not quite significant statistically. Choroidal haemorrhage is about half as frequent as subhyaloid, intraretinal, and perineural haemorrhages, indicating either that greater trauma is required for choroidal haemorrhage (as the case ranking suggests), or that the choroid is more distant from the source of trauma than the other types of haemorrhage. Vitreous haemorrhage is the least common form of intraocular haemorrhage, and was present in the two (of five) infants with highest eye trauma scores (but without choroidal haemorrhage) and probably requires the somewhat idiosyncratic rupture of the posterior hyaloid membrane.

RELATION BETWEEN CNS AND OCULAR INIURIES Our results indicate conclusively that there is a direct relation between the presence and severity of both intracerebral trauma and intraocular injuries. These various injuries can be scored and ranked in order of severity, which allows inferences to be made about the order in which the injuries are sustained with increasing severity of head injury or levels of head trauma. Thus, with increasing trauma to the head (Table 3), an infant is likely to

Table 3 Head trauma and developing central nervous system (CNS) and eye injuries in non-accidental injury in children

\begin{tabular}{lll}
\hline $\begin{array}{l}\text { Trauma } \\
\text { level }\end{array}$ & CNS injury & Eye injury \\
\hline \hline & & \\
& & \\
& & \\
& & \\
0 & None & None \\
\hline
\end{tabular}

The table indicates the types of CNS and eye injuries with increasing levels of head trauma. Note that the trauma scale is relative but not necessarily linear (point 1 represents major trauma which increases further to point 4 ), and is intended to indicate a trend only. develop subdural haemorrhage first, followed by (at slightly higher levels of trauma) subhyaloid and intraretinal haemorrhages and perineural (subdural) optic nerve sheath haemorrhage. Slightly more trauma will produce retinal detachment, and only the most severe trauma will cause choroidal and vitreous haemorrhages, which coincide with development of subarachnoid and intracerebral haemorrhages and/or cerebral lacerations. This sequence of events with increasing trauma gives some insight into the mechanism of the injuries. It is well recognised that shaking/whiplash injury to the head produces subdural haemorrhage, as the inertia of the brain (accelerating from a stationary position) or its momentum (when the moving head is subject to rapid deceleration) results in movement of the brain relative to the meninges, with resulting trauma to thin veins, and subdural haemorrhage.

With slightly more head trauma than that required for subdural haemorrhage, ocular injury occurs in the form of subhyaloid and intraretinal haemorrhage, and haemorrhage in the most anterior position of the optic nerve sheath. With further trauma, retinal detachment occurs. We believe that these four lesions can all be explained by invoking a mechanism similar to that causing subdural haemorrhage - namely, the effect of inertia/momentum of the vitreous body within the eye during cycles of acceleration/deceleration during shaking. Our findings on plotting the exact site of the retinal lesions, which show that subhyaloid haemorrhage and retinal detachment are most frequent at the periphery of the retina, and posteriorly in relation to the optic nerve, support this hypothesis, as these are the precise sites at which the vitreous is most strongly attached to the retina. ${ }^{20}$ Thus, the mass of the vitreous (which forms a large proportion of the mass of the globe) acts upon the points at which it is attached during both acceleration or deceleration, with up/down, left/right, axial or rotational movement, producing first small haemorrhages in the retina (intraretinal and subhyaloid haemorrhage) then retinal detachment (directly, with haemorrhage having an additive effect).

Optic nerve sheath haemorrhage is, we believe, the result of angular, rotational, or axial movement of the eye about a point in the most anterior part of the optic nerve, posterior to the sclera (the posterior half of the optic nerve is relatively fixed by the bony margins of the posterior orbit and by the extraocular muscles). Again, the mass of the vitreous forms the majority of the mass of the eye, and distortion and stretching of the small veins communicating between the optic nerve and its dura (sheath) are likely to be the major source of this haemorrhage.

More trauma is likely to be required to inflict the ocular injuries than the trauma needed for development of subdural haemorrhage because, although the angular momentum/angular acceleration and deceleration are identical at all sites, the size and mass of the brain (rotating about the top of the cervical 
spine) together lead to greater relative speed, forces, and distance travelled by the brain relative to meninges than vitreous relative to retina, or optic nerve relative to nerve sheath.

The pattern of sequential development and coexistence of the cerebral and ocular injuries have important clinical and prognostic implications for paediatric practice. Both ocular and central nervous system injuries such as we have described have been demonstrated in accidental and non-accidental childhood injury by Gilliland et $a l,{ }^{10}$ and we agree that, in the absence of a verifiable history of accidental trauma, the combination of life threatening CNS injuries and ocular haemorrhage are likely to be the result of child abuse, although retinal haemorrhages can be associated with a variety of other causes of raised central venous pressure including epileptic fits, asphyxia (as in our case 6), and subarachnoid haemorrhage, and can be present in normal neonates. ${ }^{21}$

In addition, however, our results give clinically important new insights into the types of lesions which may coexist in children with head injury of accidental or non-accidental aetiology. For example, an unconscious child with a combination of intraocular (vitreous, subhyaloid, intraretinal, and choroidal) haemorrhages and retinal detachment has a very high chance of intracerebral haemorrhage or lacerations in addition to subdural haemorrhage, the most common complication, and this has important implications for surgical management and prognosis. With lesser degrees of, albeit serious, head injury the earliest and most clinically apparent injuries in the eyes, subhyaloid, and/or intraretinal haemorrhage followed by small retinal detachments, may precede the onset of signs of cerebral injury. ${ }^{22}$ Our findings indicate that the most likely site for these intraocular lesions will be in the peripheral retina (which is only visible by indirect ophthalmoscopy), and that incidence of these lesions will be lower in the equatorial retina and in relation to the optic nerve (where angular momentum and vitreous attachment strengths are relatively smaller). As both subhyaloid and intraretinal haemorrhages require more trauma for their production than subdural haemorrhage, this intracranial complication should be suspected in all children where these haemorrhages or retinal detachment are present.

\section{CONCLUSIONS}

We believe that our study provides, for the first time, firm evidence that intraocular injuries in non-accidental head injury are due to the effect of vitreous traction forces, and that a similar mechanism is likely in accidental head injury accompanied by ocular injuries. The earliest intraocular lesion to accompany subdural haemorrhage in accidental or non-accidental head injury is likely to be peripheral subhyaloid haemorrhage, with or without local retinal detachment, and these peripheral retinal lesions could be missed on clinical examination if indirect ophthalmoscopic examination is omitted. At higher trauma levels intraocular haemorrhage at multiple sites combined with retinal detachment may indicate the presence of cerebral lacerations or intracranial haemorrhage in addition to subdural haemorrhage, with important clinical and prognostic implications.

1 Purtscher O. Noch unbekannte Befunde nach Schadel trauma. Ber Zusammenkunft Dtsch Ophthalmol Ges 1910; 36: 294-301.

2 Caffey J. Multiple fractures in the long bones of infants suffering from chronic subdural haematomas. $\mathrm{Am}$ Roentgenol 1946; 56: 163-73.

3 Tardieu A. Etude medico-legale sur les services et mauvais traitments exerces sur des enfants. Ann Hyg Pub Med Leg $1860 ; 13: 361-98$

4 Kempe CH, Silverman FN, Steele BF, Droegemueller W, Silver HK. The battered-child syndrome. $¥ A M A 1962$ 181: 14 .

5 Caffey J. On the theory and practice of shaking infants: its potential residual effects of permanent brain damage and mental retardation. Am $¥$ Dis Child 1972; 124: 161-9.

6 Caffey J. The whiplash infant syndrome: manual shaking by the extremities with whiplash-induced intracranial and intraocular bleeding linked with permanent brain damage and mental retardation. Paediatrics 1974; 54: 396-403.

7 Kaplan B. Ophthalmic issues in family violence and neglect. In: Albert DM, Jakobiec FA, eds. Principles and practice of ophthalmology. Philadelphia: WB Saunders, 1994; 5: 3757-62.

8 Morris DA. Ocular trauma. In: Garner A, Klintworth GK eds. Pathobiology of ocular disease. A dynamic approach. 2nd eds. Pathobiology of ocular disease. A dynamic approac

9 Eisenbrey AB. Retinal haemorrhage in the battered child. Child's Brain 1988; 5: 40-4.

10 Gilliland MGF, Luckenbach MW, Chenier TC. Systemic and ocular findings in 169 prospectively studied child deaths: retinal haemorrhages usually mean child abuse. Forensic Science International 1994; 68: 117-32.

11 Brain RB. Diseases of the nervous system. 5th ed. Oxford: Oxford University Press, 1955.

12 Gilkes MJ, Mann TP. Fundi of battered babies. Lancet 1967; 2: 468-9.

13 Greenwald MJ, Weiss A, Oesterle CS, Friendly DS Traumatic retinoschisis in battered babies. Ophthalmology 1986; 93: 618-25.

14 Duhaime A-C, Gennarelli TA, Thibault LE, Bruce DA, Margulies SS, Wiser R. The shaken baby syndrome, a clinical pathological and biochemical study. 7 Neurosurg 1987; 66: 409-15.

15 Hadley MN, Sonntag VKH, Rekate HL, Murphy A. The infant whiplash-shake injury syndrome: a clinical and pathological study. Neurosurgery 1989; 24: 536-40.

16 Kleinman PK. Diagnostic imaging in infant abuse. $A m$ Roentgenol 1990; 155: 703-12.

17 Carty H, Ratcliffe J. The shaken infant syndrome. Parents and other carers need to know of its dangers. $B M \mathcal{Y} 1995$; 310: 344-5.

18 Munger CE, Peiffer RL, Bouldin TW, Kylstra JA, Thompson RL. Ocular and associated neuropathologic observations in suspected whiplash shaken infant syndrome. A retrospective study of 12 cases. Am $\mathcal{F}$ Forensic Med Pathol 1993; 14: 193-200.

19 Budenz DL, Farber MG, Mirchandani HG, Park H, Rorke LB. Ocular and optic nerve hemorrhages in abused infants with intracranial injuries. Ophthalmology 1994; 101: 559-65.

20 Sebag J. Anatomy and pathobiology of the vitreo-retinal interface. Eye 1992; 6: 541-52.

21 Cavanagh N. Non-accidental injury. In: Taylor D, ed. Pediatric ophthalmology. Boston: Blackwell, 1990: 545-50. 22 Spaide RF, Swengel RM, Scharre DW, Mein CE. Shaken baby syndrome. Am Fam Physician 1990; 41: 1145-52. 